\title{
Heavy-flavour production in ATLAS: Onia and open flavour hadrons
}

loannis Nomidis* on behalf of the ATLAS collaboration

Carleton University, Canada

E-mail: ioannis.nomidisecern.ch

Studies of heavy-flavour production at the LHC are interesting in order to understand the direct heavy-quarkonium production mechanism and test the predictions for $b \bar{b}$ production at the new energy regime. Recent ATLAS results using the charmonium states $\chi_{c 1}, \chi_{c 2}$ and $\psi^{\prime}$ are presented. The measurements of the direct $(p p \rightarrow c \bar{c} X)$ and indirect $\left(p p \rightarrow B X \rightarrow c \bar{c} X^{\prime}\right.$ ) charmonium production extend to higher $p_{\mathrm{T}}$ than earlier measurements from CMS and LHCb, which is important in the comparison of the results with theoretical predictions from various models.

The 15th International Conference on B-Physics at Frontier Machines at the University of Edinburgh, 14 -18 July, 2014

University of Edinburgh, UK

${ }^{*}$ Speaker. 


\section{Introduction}

The LHC offers a chance for new studies of heavy-flavour production in order to test Quantum Chromo-Dynamics (QCD) calculations not only at the new energy regime, but also at higher $p_{\mathrm{T}}$ and wider rapidity range than what was studied before, thanks to the excellent coverage of the LHC detectors. The understanding of heavy quarkonia production, in particular, is still an open issue and there are quite a few models providing theoretical predictions which should be tested. Regarding heavy-quark pair production, the goal is to test the available next-to-leading-order (NLO) calculations which could describe data from the Tevatron experiments well but come with large uncertainties of up to $30 \%-40 \%$ due to renormalisation and factorisation scales, mainly, while the new experimental measurements can reach better precision.

ATLAS [1], a general-purpose detector optimised for high- $p_{\mathrm{T}}$ physics at the LHC, can contribute in the $B$-physics sector by complementing the dedicated experiment, $\mathrm{LHCb}$, with measurements in the central rapidity region. By exploiting the inner detector and the muon spectrometer which overlap in the region $|\eta|<2.5$, a variety of $B$-physics studies can be performed with the bulk of the $p p$ collision data recorded during Run-1 of the LHC (2010-2012). Results discussed in these proceedings were obtained from data recorded during 2011 with dimuon triggers. We will concentrate on the recent ATLAS publications on prompt and non-prompt production of $\chi_{c 1}$, $\chi_{c 2}$ [2] and $\psi^{\prime}$ mesons [3], obtained with $4.5 \mathrm{fb}^{-1}$ and $2.1 \mathrm{fb}^{-1}$ of data, respectively. First, general information about the experimental techniques used in the measurements is given in Section 2 and then the results are presented. Since the prompt and non-prompt production mechanisms are different in their theoretical description, the results for each one are grouped and presented separately. Results for prompt production are shown in Section 3 and compared with predictions of the non-relativistic QCD model (NRQCD, available at NLO), colour-singlet model (CSM, available at LO for $\chi_{c}$ and at NNLO* for $\psi^{\prime}$ ), $p_{\mathrm{T}}$ factorisation and colour-evaporation model (CEM). The results for non-prompt production are shown in Section 4, compared with fixed-order plus next-toleading-logarithm (FONLL) calculations and, for the case of $\psi^{\prime}$, with the NLO predictions of the general-mass variable-flavour-number scheme (GM-VFNS) calculations. ${ }^{1}$

\section{Experimental procedure}

Charmonium resonances are produced either promptly in $p p$ collisions or in decays of $B$ mesons. The two production modes (prompt and non-prompt) can be resolved by exploiting the displaced vertex of the charmonium from $B$-meson decays. Employing a two-dimensional maximum likelihood fit method using the mass and pseudo-proper decay time of the charmonium state, two physics measurements are extracted: the prompt production cross-section and the non-prompt cross-section for inclusive decays of $B$ hadrons to charmonium $(p p \rightarrow B \rightarrow c \bar{c} X)$.

In the calculation of the cross-section (times the branching ratio) the reconstruction and trigger efficiencies are extracted from the data, while the acceptance correction comes from a generator and is model dependent. It depends on the spin-momentum alignment of the charmonium which is unknown, in principle, for direct onia produced in $p p$ collisions. The usual approach followed in ATLAS is to quote results assuming the isotropic decay scenario while considering all possible

\footnotetext{
${ }^{1}$ A detailed list of references for the theoretical predictions can be found in the ATLAS papers.
} 
scenarios to define an "envelope" for the measurement. This is in fact the largest uncertainty in all direct onia production studies. For $\Upsilon$ mesons, for example, the acceptance can vary by as much as one order of magnitude at low $p_{\mathrm{T}}$ and high rapidity [4]. However, it is important to point out that this uncertainty becomes small at high $p_{\mathrm{T}}$, so this region is important to reach experimentally.

We identify $J / \psi$ mesons in the dimuon decay channel and reconstruct $\chi_{c}$ states in the $\chi_{c} \rightarrow$ $J / \psi \gamma$ decay and $\psi^{\prime}$ in the $\psi^{\prime} \rightarrow J / \psi \pi^{+} \pi^{-}$decay. In the case of $\chi_{c}$, photons are identified through their conversions in the inner detector. In the left plot (a) of Figure 1 shown is the invariant mass difference between the $\mu^{+} \mu^{-} \gamma$ and $\mu^{+} \mu^{-}$combinations; $\chi_{c 1}, \chi_{c 2}$ states stand clearly above the background and are nicely resolved. The $\chi_{c 0}$ peak is also visible but has a small yield due to the small branching to $J / \psi \gamma$ and was not considered in this analysis. The right plot (b) in the same Figure shows the pseudo-proper decay time of $\chi_{c}$ candidates. The projection of the result of the fit to both mass and decay time from which the $\chi_{c 1}$ and $\chi_{c 2}$ yields are extracted for the prompt and non-prompt fractions is also shown. The invariant mass for the reconstructed $J / \psi \pi^{+} \pi^{-}$candidates is shown in Figure 2. A clean peak of about 10k signal events is seen (along with the $\mathrm{X}(3872)$ resonance). Although this result was obtained with just half of the full 2011 data sample, there are enough statistics to split the candidates in three intervals of absolute rapidity $(0-0.75,0.75-1.5$, 1.5-2.0) and perform a differential cross-section measurement as a function of $p_{\mathrm{T}}$ in each rapidity interval. For $\chi_{c}$, the measurement is done only in the $0-0.75$ interval where the mass resolution, crucial for resolving the $\chi_{c}$ states, is optimum.

\section{Prompt production of $\chi_{c}$ and $\psi^{\prime}$ mesons}

The cross-section for $\chi_{c 1}$ and $\chi_{c 2}$ production is shown in Figures 3 (a) and 3 (b), respectively, as a function of the $p_{\mathrm{T}}$ of the $J / \psi$ meson, which allows a direct estimation of the feed-down contribution to the inclusive prompt $J / \psi$ production. The theoretical predictions for the crosssection are also plotted to compare with the measurements. At LO, CSM predictions underestimate the production, while the $p_{\mathrm{T}}$ factorisation overestimates it. Going to NLO accuracy in the NRQCD model, predictions are in good agreement with the data, indicating that the higher-order corrections are important in the case of $\chi_{c}$ production. In Ref. [2], the cross-sections are also given as function of the $p_{\mathrm{T}}$ of the $\chi_{c}$ meson; comparison with the theory predictions is also good. Therefore, within the available precision, there is good understanding of the kinematics of the $\chi_{c}$ production and decay.

As stated earlier, the relative production of $\chi_{c}$ mesons has to be measured in order to have a quantitative estimation of the feed-down contributions from the higher to the lower charmonium states. In Figure 4 (a), shown is the ratio of $\chi_{c}$ production over inclusive $J / \psi$ production, measured in bins of $J / \psi p_{\mathrm{T}}$. The measurement is in agreement with the result from LHCb [5] in the overlapping $p_{\mathrm{T}}$ region, while both measurements achieve better precision than that of the theory prediction. The right plot (b) in the same Figure shows the ratio of $\chi_{c 1}$ over $\chi_{c 2}$ production, considering also the branching to $J / \psi \gamma$ for each one. Drawn with black dots is the ATLAS measurement and with green dots is the measurement from CMS [6]; results from the two experiments are consistent. In the comparison with theory we see that CSM at LO can reproduce the $p_{\mathrm{T}}$ dependance of this ratio but fails to reproduce its value. NRQCD does a much better job but the uncertainty of the theory prediction is rather large. 


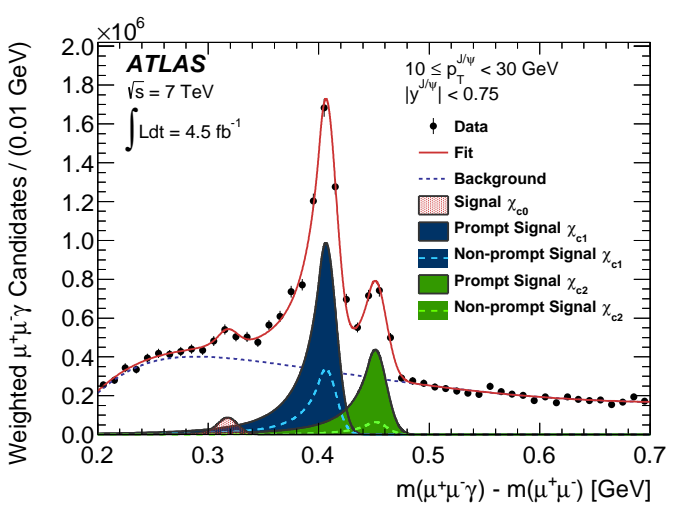

(a)

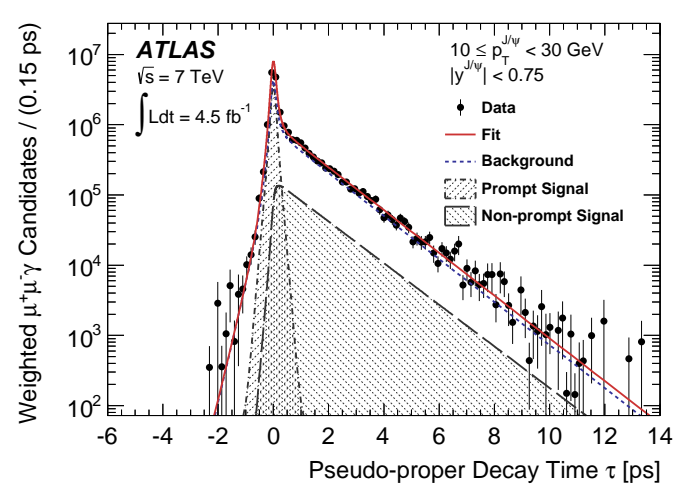

(b)

Figure 1: Distributions of the mass difference (a) and pseudo-proper decay time (b) for $\chi_{c}$ candidates, corrected for acceptance and experimental efficiency. The result of the simultaneous fit to both distributions, from which the prompt and non-prompt $\chi_{c}$ signals are extracted, is shown by the overlaid solid red lines. (Plots from Ref. [2])

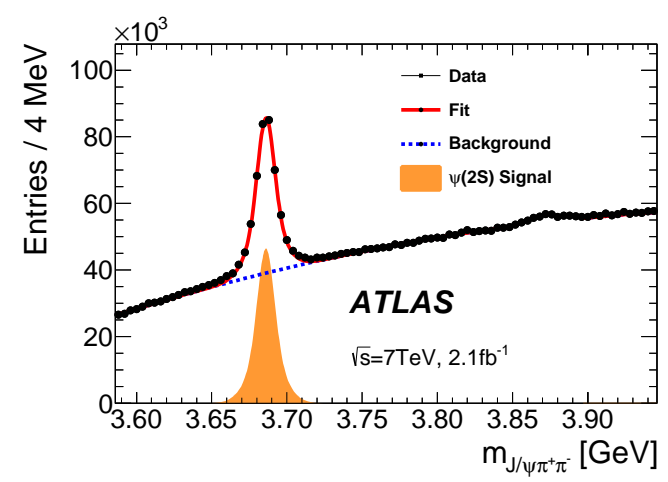

Figure 2: The $J / \psi \pi^{+} \pi^{-}$invariant mass spectrum, before corrections for acceptance and experimental efficiency, in the region between $3.586 \mathrm{GeV}$ and $3.946 \mathrm{GeV}$. The $\mathrm{X}(3872)$ resonance is also visible. (Plot from Ref. [3])

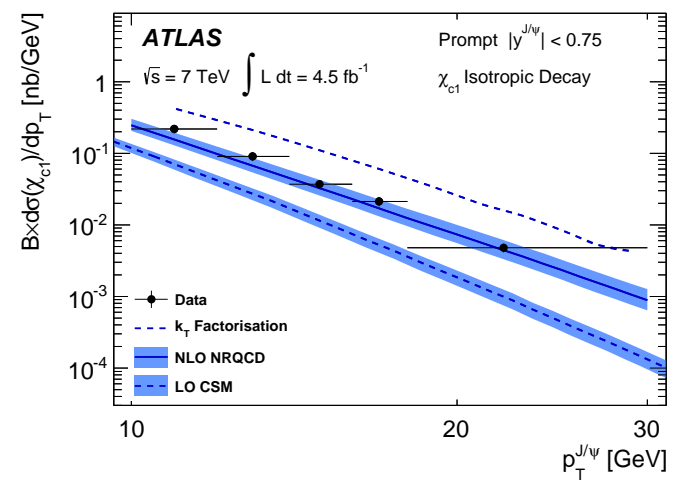

(a)

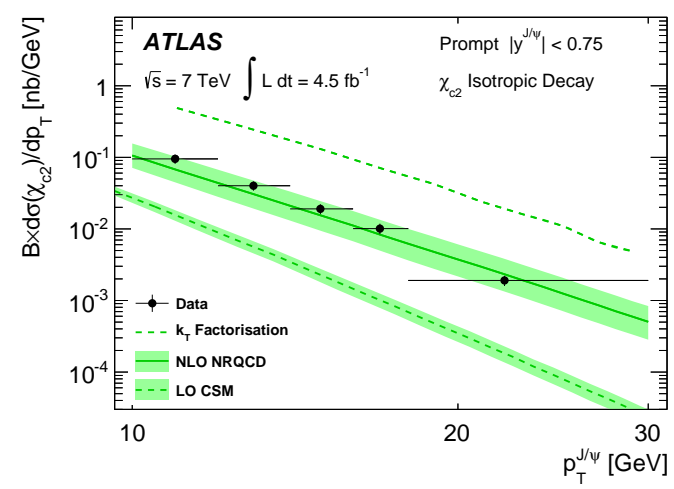

(b)

Figure 3: Differential cross-section of prompt $\chi_{c 1}$ (a) and $\chi_{c 2}$ (b) production in bins of $p_{\mathrm{T}}$ of the $J / \psi$ meson in the decay of $\chi_{c}$, along with theoretical predictions. (Plots from Ref. [2]) 


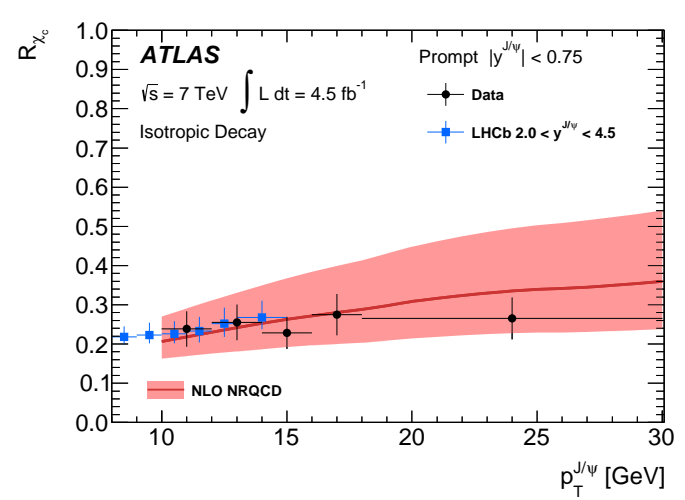

(a)

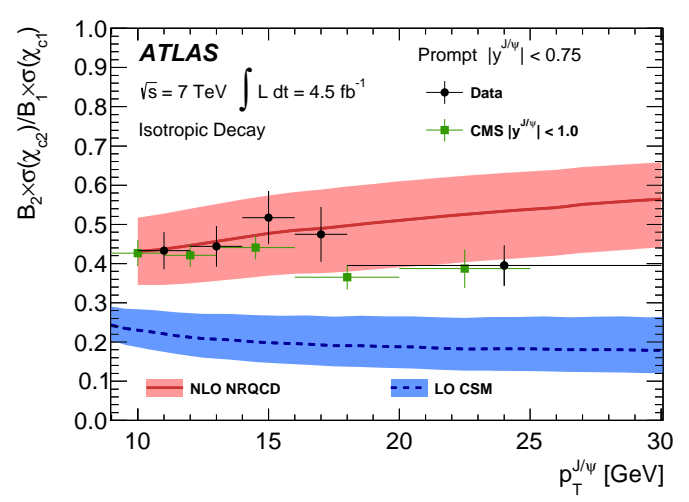

(b)

Figure 4: Shown on the left (a) is the contribution of $\chi_{c} \rightarrow J / \psi \gamma$ to the inclusive $J / \psi$ production for prompt charmonium in bins of $p_{\mathrm{T}}$ of the $J / \psi$ meson. On the right (b), the $\chi_{c 2} / \chi_{c 1}$ ratio is also given in bins of $p_{\mathrm{T}}$ of the $J / \psi$ meson. Results for LHCb and CMS are also shown for comparison in the overlapping $p_{\mathrm{T}}$ region, together with theoretical predictions. (Plots from Ref. [2])

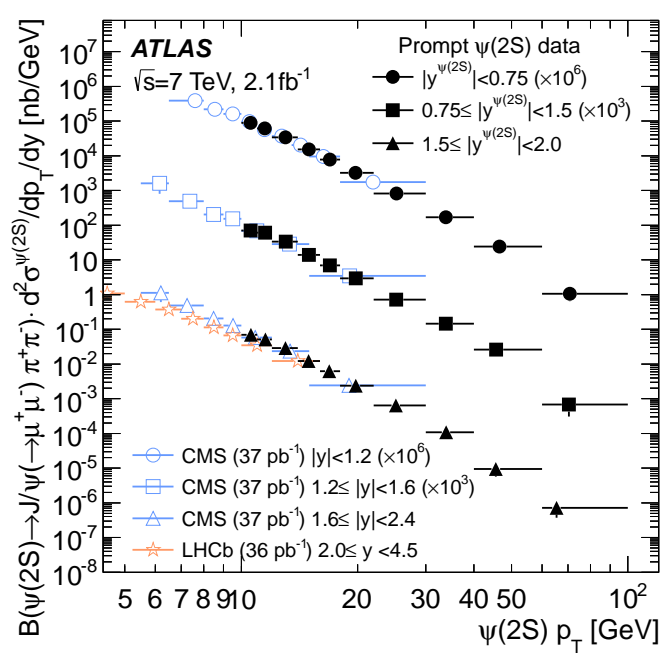

(a)

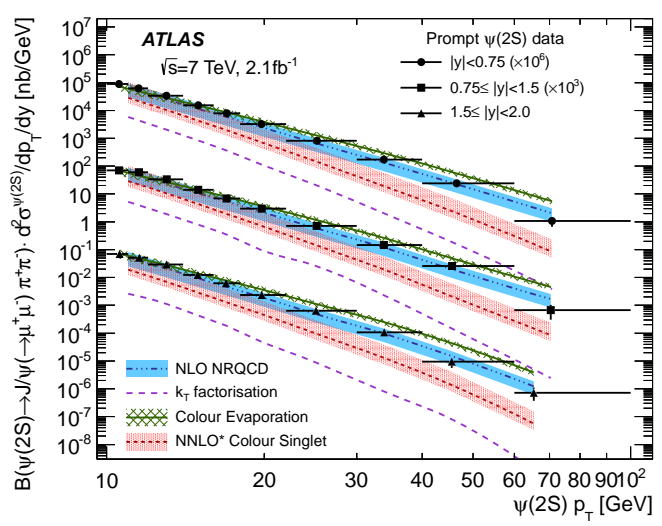

(b)

Figure 5: Differential cross-section for prompt $\psi^{\prime}$ production as a function of $p_{\mathrm{T}}$ of the $\psi^{\prime}$ meson in three rapidity intervals, as indicated on the plots. On the left (a), the experimental data from ATLAS, CMS and LHCb are superimposed. On the right (b), the ATLAS points are shown together with theoretical predictions for comparison. (Plots from Ref. [3])

The $\psi^{\prime}$ meson production is measured as a function of $p_{\mathrm{T}}$ in three rapidity intervals, covering the region $|y|<2$. The measurement is shown in Figure 5 along with experimental data from CMS [7] and $\mathrm{LHCb}$ [8] (a) and the theoretical predictions (b). Data from the experiments are consistent in the overlapping regions, with the advantage of the ATLAS measurement being that it extends to higher $p_{\mathrm{T}}$. The model that does better in describing the data is NRQCD. The CEM and the NNLO* CSM calculations cannot reproduce the shape. The $p_{\mathrm{T}}$ factorisation calculations underestimate the production significantly (oppositely to what is seen in the $\chi_{c}$ results). 


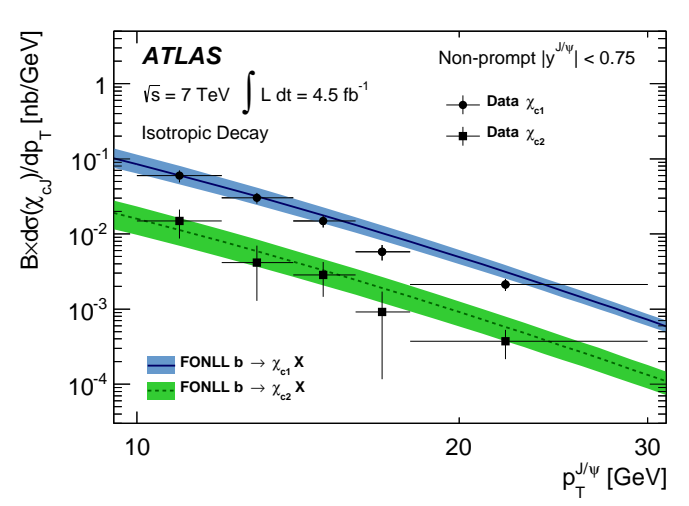

(a)

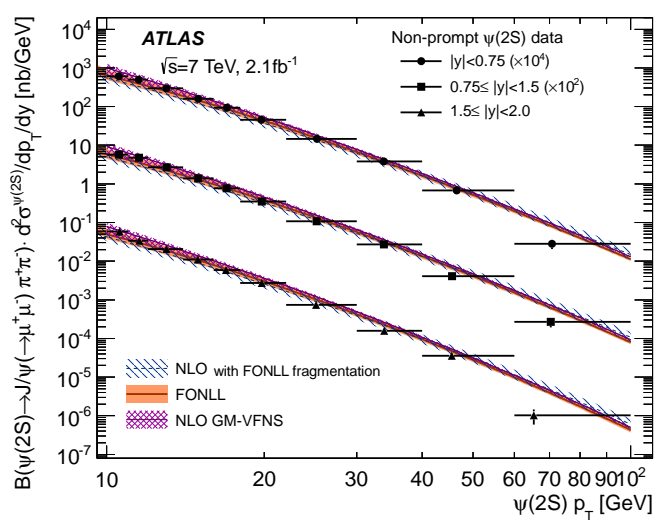

(b)

Figure 6: Differential cross-section for non-prompt $\chi_{c 1}$ and $\chi_{c 2}$ production (a) and $\psi^{\prime}$ production (b) as a function of $p_{\mathrm{T}}$. Theoretical predictions are also shown for comparison. (Plots from Ref. [2] and [3])

\section{Non-prompt production of $\chi_{c}$ and $\psi^{\prime}$ mesons}

The cross-section for $\chi_{c 1}$ and $\chi_{c 2}$ production is shown as function of $p_{\mathrm{T}}$ in Figure 6 (a) and is compared to calculations obtained with the FONLL framework. The predictions compare nicely with the data within the uncertainties, experimental and theoretical. The relative production of the non-prompt $\chi_{c}$ states is also measured and the results can be found in Ref. [2]. In the same paper you can also find a measurement of the branching fraction for $B^{+} \rightarrow \chi_{c 1} K^{+}$which is in excellent agreement with the world average and is the best measurement at hadron colliders so far. This measurement supports that in this analysis systematics are well under control.

The $\psi^{\prime}$ cross-sections are shown in Figure 6 (b) and allow comparisons with theoretical predictions up to high $p_{\mathrm{T}}$ of about $70 \mathrm{GeV}$ in three rapidity intervals in the range $|y|<2$. As for $\chi_{c}$, calculations obtained with the FONLL framework can describe the data very well. Predictions obtained by using NLO calculation together with the fragmentation functions from the FONLL framework are also shown, demonstrating the improvement that the FONLL calculations provide, especially regarding the uncertainty of the calculations at high $p_{\mathrm{T}}$. The NLO GM-VFNS predictions do a very good job describing the data as well and their associated theoretical uncertainties are even smaller. Focusing on the comparison of the data to the theory predictions at high $p_{\mathrm{T}}, \mathrm{a}$ tendency of the predictions to overshoot the data is seen. This is similar to what was observed in the $B \rightarrow J / \psi X$ measurement [9]. However, there is good agreement at high $p_{\mathrm{T}}$ when comparing FONLL predictions for charged $B$-meson production with measurements in the fully reconstructed decay $B^{ \pm} \rightarrow J / \psi K^{ \pm}$[10]. This indicates a possible mismodelling in $B$-hadron composition and decay kinematics, rather than in the $b$-quark fragmentation model in FONLL.

\section{Conclusions}

These proceedings presented recent ATLAS measurements of the cross-sections for $\chi_{c 1}, \chi_{c 2}$ and $\psi^{\prime}$ states produced promptly and in decays of $B$ hadrons in $p p$ collisions at $7 \mathrm{TeV}$ at the LHC. 
The prompt $\chi_{c}$ and $\psi^{\prime}$ results, together with the earlier measurement of ATLAS for the inclusive prompt $J / \psi$ production, comprise a complete list of studies of charmonium production in order to disentangle the various effects that make the theoretical interpretation of the experimental results difficult. The prompt $\chi_{c}$ measurements provide an estimation of the feed-down contributions to the $J / \psi$ production, and the prompt $\psi^{\prime}$ measurements extend to high $p_{\mathrm{T}}$ where the uncertainty due to the unknown spin-momentum alignment is small. In all cases, the NRQCD (Colour-Octet) model at NLO accuracy does best describing the data. The final conclusion, however, can be drawn only after taking into consideration spin-momentum alignment measurements; an ATLAS measurement for this is highly anticipated.

As seen also in earlier results from ATLAS, CMS and LHCb for non-prompt production, the cross-section measurements are in agreement with the predictions of FONLL calculations. For the $\psi^{\prime}$ measurement that was presented here and extends to higher $p_{\mathrm{T}}$ than what measured by CMS and LHCb, we see good agreement with both FONLL and GM-VFNS predictions. We observe a tendency of the theoretical calculations to predict a slightly harder spectrum than what seen in data.

\section{References}

[1] ATLAS Collaboration, JINST 3 (2008) S08003.

[2] ATLAS Collaboration, JHEP 07 (2014) 154

[3] ATLAS Collaboration, JHEP 09 (2014) 079

[4] ATLAS Collaboration, Phys. Rev. D 87, 052004 (2013)

[5] LHCb Collaboration, Phys. Lett. B 718 (2012) 431

[6] CMS Collaboration, Eur. Phys. J. C 72 (2012) 2251

[7] CMS Collaboration, JHEP 02 (2012) 11

[8] LHCb Collaboration, Eur. Phys. J. C 72 (2012) 2100

[9] ATLAS Collaboration, Nucl. Phys. 458 B850 (2011)

[10] ATLAS Collaboration, JHEP 10 (2013) 042 